\title{
On strategy-proof social choice correspondences
}

\section{Shin Sato}

Published online: 28 February 2008

(C) Springer-Verlag 2008

\section{Erratum to: Soc Choice Welfare DOI 10.1007/s00355-007-0285-5}

I am grateful to Carmelo Rodríguez-Álvarez for pointing out my error in Sato (2007). In Sect. 3, the definition of $\succsim^{\min }$ and $\succsim^{\max }$ should be changed as follows: For $A=$ $\left\{a_{1}, \ldots, a_{k}\right\}$ and $B=\left\{b_{1}, \ldots, b_{h}\right\}$,

- assume $a_{k} P a_{k-1} P \cdots P a_{2} P a_{1}$ and $b_{h} P b_{h-1} \cdots P b_{2} P b_{1}$, then

$$
\begin{aligned}
& A \succsim^{\min } B \\
& \Longleftrightarrow\left\{\begin{array}{c}
\exists l \leq(\min \{k, h\}+1) / 2:\left[\begin{array}{c}
a_{l} P b_{l} \text { or }\left[\begin{array}{c}
a_{l}=b_{l} \\
a_{k-l+1} P b_{h-l+1}
\end{array}\right] \\
a_{m}=b_{m}, a_{k-m+1}=b_{h-m+1} \forall m<l
\end{array}\right] \\
\text { or } \\
{\left[\begin{array}{l}
k \geq h \leq(h+1) / 2,\left[\begin{array}{r}
a_{m}=b_{m} \\
a_{k-m+1}=b_{h-m+1}
\end{array}\right]
\end{array}\right]}
\end{array}\right.
\end{aligned}
$$

The online version of the original article can be found under doi:10.1007/s00355-007-0285-5.

S. Sato $(\varangle)$

Graduate School of Economics, Keio University,

2-15-45, Mita, Minato-ku, Tokyo, Japan

e-mail: sato@gs.econ.keio.ac.jp 
- assume $a_{1} P a_{2} P \ldots P a_{k-1} P a_{k}$ and $b_{1} P b_{2} P \ldots P b_{h-1} P b_{h}$, then

$$
\begin{aligned}
A \succsim^{\max } B \\
\qquad l \leq(\min \{k, h\}+1) / 2:\left[\begin{array}{c}
a_{l} P b_{l} \text { or }\left[\begin{array}{c}
a_{l}=b_{l} \\
a_{k-l+1} P b_{h-l+1}
\end{array}\right] \\
a_{m}=b_{m}, a_{k-m+1}=b_{h-m+1} \quad \forall m<l
\end{array}\right] \\
\text { or } \left.\begin{array}{c}
k \leq h \\
\forall m \leq(k+1) / 2,\left[\begin{array}{c}
a_{m}=b_{m} \\
a_{k-m+1}=b_{h-m+1}
\end{array}\right]
\end{array}\right]
\end{aligned}
$$

Accordingly, the following changes are needed.

Example 3.1 On $\mathcal{A}, \succsim^{\max }$ does not coincide with $\succsim^{\text {top }}$. $\succsim^{\max }$ is such that $\left\{x_{1}, x_{5}\right\} \succ^{\max }$ $\left\{x_{1}, x_{4}, x_{5}\right\} \succ^{\max }\left\{x_{2}, x_{3}, x_{5}\right\} \succ^{\max }\left\{x_{3}\right\}$ whereas $\succsim^{\text {top }}$ is such that $\left\{x_{1}, x_{4}, x_{5}\right\} \succ^{\text {top }}$ $\left\{x_{1}, x_{5}\right\} \succ^{\text {top }}\left\{x_{2}, x_{3}, x_{5}\right\} \succ^{\text {top }}\left\{x_{3}\right\}$. (This change does not affect the other parts of the example.)

Proof of Theorem 3.2 The proof is similar to the proof of Theorem 3.1. To show that $x_{2} \in F\left(R_{N}\right)$, we derive a contradiction to $x_{2} \notin F\left(R_{N}\right)$ as follows: if $\left\{x_{1}, x_{|X|}\right\}=$ $F\left(R_{N}\right)$, then $F\left(R_{2}^{\prime}, R_{-2}\right) \succ_{2}^{\min } F\left(R_{N}\right)$. Otherwise, $F\left(R_{2}^{\prime}, R_{-2}\right) \succ_{2}^{\max } F\left(R_{N}\right)$. These are contradictions to $E^{B P X}$ strategy-proofness. 